\title{
Canals under sediment-based ice sheets
}

\author{
FeLix S. L. NG \\ Mathematical Institute, University of Oxford, Oxford OX1 3LB, England
}

\begin{abstract}
Classical theories of channelized subglacial drainage identify a melting vs creep balance at the channel-ice boundary that is crucial in determining its steady flow properties, but this consideration neglects the role of erodible-deformable basal sediments, especially in controlling channel morphology. Here we present a mathematical model for channels underneath ice sheets, which incorporates the detailed mechanics of sediment transport over a mobile till-bed interface, and which allows a variable, wide channel cross section also. The resulting drainage conditions depend on the sediment flux $(q)$ as well as the water flux $(Q)$ through the channel - these quantities being controlled by the rates at which sediment and meltwater are captured by the flow. An approximate analytical solution indicates a "canal"-type drainage law (Walder and Fowler, 1994) of the form $N_{\mathrm{c}}^{n} \propto \Psi^{-1} Q^{-5 / 2} q^{3 / 2}$, where $N_{\mathrm{c}}$ is the effective channel pressure, $n$ is the exponent in Glen's flow law for ice, and $\Psi$ is the imposed hydraulic gradient (due to topography). By solving the downstream problem numerically, the canal characteristic has been confirmed and found to be insensitive to upstream conditions. Thus, low effective pressures can result from high basal water flux or melt rates. This supports the contention that canals may be a central component in fast ice flow over soft beds. The proposed model also establishes a fundamental link between the plumbing system of a glacier and its sediment budget.
\end{abstract}

\section{INTRODUCTION}

The presence of sediments underneath glaciers has far reaching consequences for their dynamics. Where ice overrides a wet subglacial till, enhanced flow velocities may result from pervasive till deformation or sliding at the ice-till interface. Evidence for these mechanisms has been obtained both in the field (Alley and others, 1986, 1987; Iverson and others, 1995) and in the laboratory (Kamb, 1991). Their operation generally relies on the water pressure at the base being high, close to the overburden pressure of the ice above. More particularly, there have been attempts to explain ice-stream formation and the (possible) surging behaviour of ice sheets in terms of a feedback instability based on these mechanisms (Fowler and Johnson, 1995, 1996; Fowler and Schiavi, 1998). The condition for instability is that the basal-water pressure increases with water flux. A crucial question is then: can drainage over a soft bed exhibit this property?

An earlier theory developed by Walder and Fowler (1994) suggests that this is the case. Given the large size of ice sheets, water flow through the till or a subglacial aquifer is usually unable to evacuate all the basal meltwater (Shoemaker, 1986; Alley, 1989); some kind of flow at the ice-till interface is therefore necessary. What Walder and Fowler have demonstrated is that for a soft bed, a network of channels can exist, each with water pressure, $p_{\mathrm{c}}$, increasing with discharge, $Q$, or equivalently effective pressure, $N_{\mathrm{c}}$, decreasing with $Q$. $\left(N_{\mathrm{c}}=p_{\mathrm{i}}-p_{\mathrm{c}}\right.$ is the difference between the overburden ice pressure and water pressure.) They refer to such channels as "canals". Overall, the subglacial interface is assumed to adopt a similar pressure-flux relationship, because of the distributed nature of the canal network.

Walder and Fowler's (1994) drainage law $N_{\mathrm{c}} \propto Q^{\beta}$, $\beta<0$, originates from an equilibrium consideration, much in the same way as in the classical theory of ice channels (Röthlisberger, 1972). However, there are two main problems with their canal model: it lacks a detailed description of how the flow interacts with till sediments and also neglects the effect of downstream variation.

In this paper, both of these constituents are shown to be important in governing the channel characteristic, and accordingly we propose an improved model. In comparison to Walder and Fowler's (1994), the theory presented here is more complete, not only because it places emphasis on sediment transport, but also because our governing equations can describe the downstream distribution of effective pressure, as in the classical model. We find that a canal-type drainage law is still possible, but determination of effective pressure now requires knowledge of sediment flux as well as discharge through the channel. This result holds wider implications for the structure of soft-bed drainage systems and the transport of subglacial sediments, and thus also for the long-term dynamics of ice sheets.

The organization of this paper is as follows. We begin by describing our conceptual model (section 2). The governing equations are then introduced and analysed, and we conduct a numerical simulation relevant for an ice sheet (section 3). This is followed by discussion (section 4). Due to the limited space, only the essentials and preliminary results of our investigation will be covered. A detailed theory of canals and further justification of the model will be given elsewhere $(\mathrm{Ng}$, manuscript in preparation).

\section{EQUILIBRIUM SOFT-BED GHANNEL}

Consider a single channel located at the ice-till interface, 
carrying a discharge $Q(s)$ and sediment volume flux $q(s)$, where $s$ is downstream distance (see Fig. la). Initially, let us suppose that the channel cross section is wide and shallow, with a variable aspect ratio (Fig. 1b). This presumption greatly simplifies our mathematical model and is consistent with the solution obtained later. We also impose (in a distributed sense) an inflow of meltwater and sediment to the channel; the supply rates are denoted respectively by $\mathcal{M}(s)$ and $\mathcal{E}(s)$. We can therefore mimic the effect of till percolation and adjoining subglacial or englacial tributaries, without having to specify the drainage-network structure.

Our model is based on the condition of equilibrium drainage, which requires the upper and lower boundaries of the channel to remain stationary. At the roof, this is achieved by a dynamic balance between creep closure and melting of the ice (Röthlisberger, 1972); whereas at the bed, a greater number of constituent processes are involved. These include (1) erosion and (2) deposition of sediment, (3) lateral and (4) downstream sediment transport, and (5) creep motion of till into the channel. Generally, the till would be characterized by a heterogeneous grain-size distribution, so sediment transport may take place as bedload and suspended load as well and this complicates the situation. For convenience, we assume here a till which consists of sufficiently fine-grained, uniform material, such that under typical conditions (when the water flux is not too small), suspension would dominate and bedload may be neglected. A more complete formulation will be described elsewhere ( $\mathrm{Ng}$, manuscript in preparation).

The crux of the model is how the mobile bed interacts with the flow and this depends on the relative importance of the five processes listed above (Fig. 1b.) Given a wide channel cross section, lateral sediment transport will be negligible because cross-stream gradients are small. The primary balance is then between sediment erosion and deposition. Essentially, this balance dictates the amount of suspended-sediment within the flow section. An analogous situation is found in alluvial river channels (Parker, 1978).

More specifically, lateral transport is relevant only in determining the bed position, but not the flow depth $(\mathrm{Ng}$, manuscript in preparation); and the precise bed-balance is that between net erosion (i.e. erosion minus deposition) and increep of the till. Under typical drainage conditions, the rates of erosion and deposition will be roughly equal and far exceed their difference. Thus, although the creep velocity may be non-zero, its effect on the suspended-sediment concentration locally (within the cross section) will be negligible.

Having said this, the cumulative effect of till creep is important in the downstream direction. This is because suspended sediment is carried along by the flow. Consider the case where tributary supply is absent: $\mathcal{E}(s)=0$ (Fig. la). At equilibrium, the observed sediment flux at any station along the channel must then be equal to the total creep flux integrated over the channel length, measured from its source to the station. Similarly, the roof is maintained by ice melting and closure, but the meltwater thus generated is advected downstream. The implication is that (at equilibrium) the channel geometry must vary downstream, in such a way that both the water and sediment phases are conserved, while local dynamic balance is maintained. As we shall see, these requirements provide sufficient constraints for determining the entire channel geometry and also the corresponding drainage characteristic. We provide a quantitative description of these ideas in the next section. $\mathbf{a}$
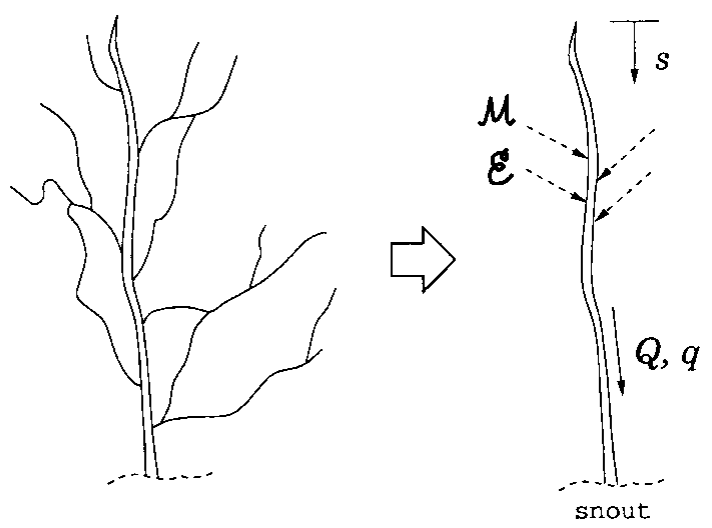

b

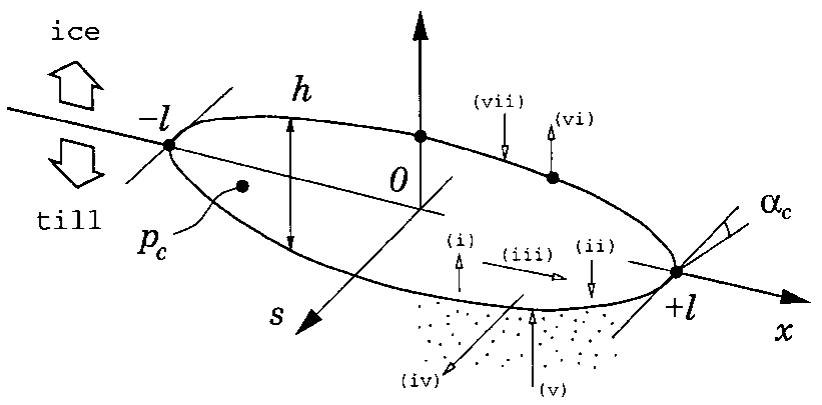

Fig. 1. Idealized model of the subglacial channel and mathematical notations used to characterize its (a) downstream and ( $b$ ) cross-sectional properties. In ( $b$ ), the channel boundaries evolve as a result of ( $i$ ) erosion, (ii) deposition, (iii) lateral transport, (iv) downstream transport of sediment; (v) creep motion of the till; (vi) melting; and (vii) viscous closure of the ice.

\section{MATHEMATIGAL MODEL}

\section{Cross-sectional processes}

We define a channel of depth $h(x, s)$ symmetrical in $x$, where $x$ and $s$ denote respectively its cross-stream and downstream coordinates (Fig. lb). Hence, if $l(s)$ is the half-width of the channel, then $h>0$ in $|x|<l$ and $h(x= \pm l)=0$. In addition we have $h / l \ll 1$, since a "wide" cross section has been assumed. In this case, suitable equations to describe the local balances (in $x$ ) are

$$
\begin{aligned}
& \frac{\hat{m}_{\mathrm{i}}}{\rho_{\mathrm{i}}}=\hat{w}_{\mathrm{i}}, \\
& \frac{v_{\mathrm{s}}(E-D)}{1-n_{\mathrm{S}}}=\hat{w}_{\mathrm{s}},
\end{aligned}
$$

in which $\hat{m}_{\mathrm{i}}$ is the melt rate of ice (mass rate per unit width per unit length of channel), $\hat{w}_{\mathrm{i}}$ and $\hat{w}_{\mathrm{s}}$ are respectively the creep-closure velocities at the roof and the bed, and $E$ and $D$ are coefficients which describe the erosion and deposition rates of sediment into/from the flow. Specifically, $v_{\mathrm{s}}(E-D)$ represents the rate of net erosion, where $v_{\mathrm{s}}$ is the (constant) grain-settling velocity. The other constants are density of ice $\rho_{\mathrm{i}}$ and bed porosity $n_{\mathrm{s}}$. Note that Equations (1) and (2) apply in the "upward" direction, normal to the $x$ and $s$ axes.

Melting is due to the heat dissipated by the turbulent flow. Given that the channel is inclined (downstream) at 
an angle, $\alpha_{\mathrm{c}}$, and has a water pressure, $p_{\mathrm{c}}$, the hydraulic gradient driving the flow is defined by

$$
-\frac{\mathrm{d} \phi}{\mathrm{d} s}=\rho_{\mathrm{w}} g \sin \alpha_{\mathrm{c}}-\frac{\mathrm{d} p_{\mathrm{c}}}{\mathrm{d} s},
$$

where $\phi$ is the water potential, $\rho_{\mathrm{w}}$ is density of water and $g$ is gravitational acceleration. In the following, we use the abbreviation $\Phi=-\mathrm{d} \phi / \mathrm{d} s$ for the hydraulic gradient, and also we introduce the effective channel pressure $N_{\mathrm{c}}\left(=p_{\mathrm{i}}-p_{\mathrm{c}}\right)$ by rewriting Equation (3) as

$$
\Phi=\Psi+\frac{\mathrm{d} N_{\mathrm{c}}}{\mathrm{d} s},
$$

in which $\Psi$ is a "basic" hydraulic gradient, imposed externally by topography. A useful approximation is $\Psi=\rho_{\mathrm{i}} g \sin \alpha_{\mathrm{s}}$, where $\sin \alpha_{\mathrm{s}}$ is the ice-surface slope (Walder and Fowler, 1994). For an ice sheet $\sin \alpha_{\mathrm{s}} \sim 10^{-3}$, so $\Psi \sim 10 \mathrm{~kg} \mathrm{~m}^{-2} \mathrm{~s}^{-2}$.

Since the flow is wide, the upper and lower boundaries may be considered to be parallel, and its thermomechanics may be summarized by the model

$$
\begin{aligned}
& \tau=\Phi h, \\
& \tau=\frac{1}{8} f \rho_{\mathrm{w}} \hat{u}^{2}, \\
& \tau \hat{u}=\hat{m}_{\mathrm{i}} L
\end{aligned}
$$

(Fowler and $\mathrm{Ng}, 1996)$. Here, $\tau$ is the total shear stress exerted at the roof and the bed, $\hat{u}$ is the depth-averaged flow velocity, $f$ is a (constant) friction factor, and $L$ is latent heat. These equations respectively represent a local force balance, a friction parameterization and local energy balance. Typically $f \sim 0.1$, by analogy to rivers (Richards, 1982). Solving for $\hat{m}_{\mathrm{i}}$ and $\hat{u}$ in these equations, we obtain

$$
\hat{m}_{\mathrm{i}}=\frac{(2 \Phi)^{3 / 2}}{L \sqrt{\rho_{\mathrm{w}} f}} h^{3 / 2}, \quad \hat{u}=\sqrt{\frac{8 \Phi}{\rho_{\mathrm{w}} f}} h^{1 / 2} .
$$

Our prescription for $E$ and $D$ is based on modelling studies in river mechanics. For wide channels, Parker (1978) proposed the expressions

$$
E=0.092\left(\frac{\tau}{2 \Delta \rho g D_{\mathrm{s}}}\right)^{3 / 2}, \quad D=\frac{v_{\mathrm{s}}}{\epsilon} \zeta,
$$

where $\Delta \rho$ is the density difference $\rho_{\mathrm{s}}-\rho_{\mathrm{w}}\left(\rho_{\mathrm{s}}\right.$ is sediment density), and $D_{\mathrm{s}}$ is the representative sediment grain-size. $\zeta$ is the total suspended-sediment content - this is the volumetric sediment concentration (a dimensionless ratio) integrated over the vertical flow column, so it is a length with unit $\mathrm{m}$. The eddy diffusivity $\epsilon$ is a (local, depth-averaged) measure of the turbulent-flow intensity; under the parallelflow formulation as used in Equations (5)-(7), an appropriate expression is

$$
\epsilon=\sqrt{\frac{\Phi f}{8 \rho_{\mathrm{w}}}} h^{3 / 2}
$$

We assume non-linear rheology for both ice and till. If we adopt Glen's law for ice (see Nye, 1953) and the constitutive law proposed by Boulton and Hindmarsh (1987) for sediment, then approximate relations for the closure rates are

$$
\hat{w}_{\mathrm{i}}=\frac{A_{\mathrm{I}} N_{\mathrm{c}}^{n}}{2} \sqrt{l^{2}-x^{2}}, \quad \hat{w}_{\mathrm{s}}=\frac{A_{\mathrm{T}} N_{\mathrm{c}}^{a}}{2 N_{\infty}^{b}} \sqrt{l^{2}-x^{2}},
$$

where $N_{\infty}$ is the effective pore-water pressure in the till, far from the channel (see $\mathrm{Ng}, 1997 ; \mathrm{Ng}$ and Fowler, submitted). We use the flow-law constants $A_{\mathrm{I}}=2 \times 10^{-24} \mathrm{~Pa}^{-3} \mathrm{~s}^{-1}, n=3$, $A_{\mathrm{T}}=3 \times 10^{-5} \mathrm{~Pa}^{\mathrm{b}-\mathrm{a}} \mathrm{s}^{-1}, a=1.33$ and $b=1.8$.
Various authors have recently questioned the validity of Boulton and Hindmarsh's (1987) viscous law for till (e.g. Kamb, 1991; Iverson and others, 1998). However, the precise form for $\hat{w}_{\mathrm{s}}$ is of minor importance in our model. Notably our concept of downstream sediment conservation (see later) is unaltered so long as the till can deform; also, we argue here that creep effects are generally negligible in the bed balance. To do this, we use conservative estimates together with the fact that Equation $(11)_{2}$ is already an upper-bound expression, based on an infinitely deep till ( $\mathrm{Ng}$ and Fowler, submitted). Suppose $N_{\infty}$ is not too small, say, $\gtrsim 0.1$ bar. Then taking $D_{\mathrm{s}}=0.05 \mathrm{~mm}, v_{\mathrm{s}}=0.05 \mathrm{~m} \mathrm{~s}^{-1}$ and the prospective values $N_{\mathrm{c}} \approx 5$ bar, $h=0.1 \mathrm{~m}, l=10 \mathrm{~m}$, it is easy to show that $\hat{w}_{\mathrm{s}} \ll v_{\mathrm{s}} E$. Thus henceforth, we neglect the creep term from Equation (2), writing

$$
E=D
$$

(This approximation is justified by our solution later.) Substitution of Equations (5), (8) $1,(9),(10)$ and (11) 1 into Equations (1) and (12) leads to

$$
\begin{aligned}
& \frac{(2 \Phi h)^{3 / 2}}{\rho_{\mathrm{i}} L \sqrt{\rho_{\mathrm{w}} f}}=\frac{A_{\mathrm{I}} N_{\mathrm{c}}^{n}}{2} \sqrt{l^{2}-x^{2}}, \\
& \zeta=\frac{0.092 \Phi^{2} h^{3}}{8 v_{\mathrm{s}}\left(\Delta \rho g D_{\mathrm{s}}\right)^{3 / 2}} \sqrt{\frac{f}{\rho_{\mathrm{w}}}}
\end{aligned}
$$

These equations provide the equilibrium depth and sediment distribution across the channel cross section, given $\Phi$, $N_{\mathrm{c}}$ and $l$ (which are functions of $s$ only).

\section{Downstream processes}

Following our description in section 2, the downstream increment of discharge $Q$ is due to ice-melting and tributary input; similarly, the increment of suspended-sediment flux, $q$, is due to sediment entering the channel via creep motion of the till and from tributaries. The corresponding massconservation equations are

$$
\begin{aligned}
& \frac{\mathrm{d} Q}{\mathrm{~d} s}=\frac{1}{\rho_{\mathrm{w}}} \int_{-l}^{l} \hat{m}_{\mathrm{i}} \mathrm{d} x+\mathcal{M}(s), \\
& \frac{\mathrm{d} q}{\mathrm{~d} s}=\left(1-n_{\mathrm{s}}\right) \int_{-l}^{l} \hat{w}_{\mathrm{s}} \mathrm{d} x+\mathcal{E}(s),
\end{aligned}
$$

in which $\mathcal{M}$ and $\mathcal{E}$ denote the distributed supply rates (to be prescribed). The appropriate flux definitions are

$$
Q=\int_{-l}^{l} h \hat{u} \mathrm{~d} x, \quad q=\int_{-l}^{l} \zeta \hat{u} \mathrm{~d} x .
$$

Our model is now completed by substituting for $\hat{m}_{\mathrm{i}}, \hat{u}, \hat{w}_{\mathrm{s}}, h$ and $\zeta$ from Equations (8), (11) $)_{2},(13)$ and (14). At this point, it is algebraically convenient also to scale the model. This is described next.

\section{Non-dimensionalization}

The head of our channel is taken at $s=0$, and its downstream (snout) end at $s=s_{0}$, where $s_{0}$ is the total channel length. By choosing the distance scale to be $[s]=s_{0}$, we can define a suitable dimensionless distance variable

$$
s^{\star}=s /[s] .
$$


Similarly, let us re-scale the other variables in the model by assigning

$$
\begin{aligned}
& {[h]=h_{0}, \quad[x]=[l]=l_{0}, \quad[\zeta]=\zeta_{0},} \\
& {[\Phi]=[\Psi]=\Psi_{0}, \quad\left[N_{\mathrm{c}}\right]=\left[N_{\infty}\right]=N_{0}, \quad[Q]=Q_{0},} \\
& {[q]=q_{0}, \quad[\mathcal{M}]=Q_{0} / s_{0}, \quad[\mathcal{E}]=q_{0} / s_{0} .}
\end{aligned}
$$

If we impose the scale relations

$$
\begin{aligned}
& \frac{4 \sqrt{2}\left(\Psi_{0} h_{0}\right)^{3 / 2}}{\rho_{\mathrm{i}} L \sqrt{\rho_{\mathrm{w}} f}}=A_{\mathrm{I}} N_{0}^{n} l_{0}, \\
& \zeta_{0}=\frac{0.092 \Psi_{0}^{2} h_{0}^{3}}{v_{\mathrm{s}}\left(2 \Delta \rho g D_{\mathrm{s}}\right)^{3 / 2}} \sqrt{\frac{f}{\rho_{\mathrm{w}}}}, \\
& Q_{0}=\sqrt{\frac{8 \Psi_{0}}{\rho_{\mathrm{w}} f}} h_{0}^{3 / 2} l_{0}, \quad q_{0}=\sqrt{\frac{8 \Psi_{0} h_{0}}{\rho_{\mathrm{w}} f}} \zeta_{0} l_{0},
\end{aligned}
$$

then, on non-dimensionalizing Equations (4) and (13)-(17), we obtain (dropping the asterisks ${ }^{\star}$ )

$$
\begin{aligned}
& \Phi=\Psi+\nu \frac{\mathrm{d} N_{\mathrm{c}}}{\mathrm{d} s}, \\
& h=\frac{N_{\mathrm{c}}^{2 n / 3}}{\Phi}\left(l^{2}-x^{2}\right)^{1 / 3}, \\
& \zeta=\frac{\Phi^{2} h^{3}}{2 \sqrt{2}}\left[=\frac{N_{\mathrm{c}}^{2 n}\left(l^{2}-x^{2}\right)}{2 \sqrt{2} \Phi}\right], \\
& \frac{\mathrm{d} Q}{\mathrm{~d} s}=\tilde{\epsilon} r \Phi^{3 / 2} \int_{-l}^{l} h^{3 / 2} \mathrm{~d} x+\mathcal{M}, \\
& \frac{\mathrm{d} q}{\mathrm{~d} s}=\frac{\tilde{\kappa} \pi\left(1-n_{\mathrm{s}}\right) N_{\mathrm{c}}^{a} l^{2}}{2 N_{\infty}^{b}}+\mathcal{E}, \\
& Q=\Phi^{1 / 2} \int_{-l}^{l} h^{3 / 2} \mathrm{~d} x, \\
& q=\Phi^{1 / 2} \int_{-l}^{l} h^{1 / 2} \zeta \mathrm{d} x .
\end{aligned}
$$

The parameters are given by

$$
\begin{aligned}
& \nu=\frac{N_{0}}{s_{0} \Psi_{0}}, \quad \tilde{\epsilon}=\frac{\Psi_{0} s_{0}}{\rho_{\mathrm{i}} L}, \\
& r=\frac{\rho_{\mathrm{i}}}{\rho_{\mathrm{w}}}, \quad \tilde{\kappa}=\frac{A_{\mathrm{T}} N_{0}^{a-b} l_{0}^{2} s_{0}}{2 q_{0}} .
\end{aligned}
$$

In the following, we assume the constants $\rho_{\mathrm{i}}=900 \mathrm{~kg} \mathrm{~m}^{-3}$, $\rho_{\mathrm{w}}=10^{3} \mathrm{~kg} \mathrm{~m}^{-3}, \rho_{\mathrm{s}}=2.65 \times 10^{3} \mathrm{~kg} \mathrm{~m}^{-3}, g=9.8 \mathrm{~m} \mathrm{~s}^{-2}, L=$ $333.5 \mathrm{~kJ} \mathrm{~kg}^{-1}, f=0.1, n_{\mathrm{s}}=0.3, D_{\mathrm{s}}=5 \times 10^{-5} \mathrm{~m}$ (coarse silt) and $v_{\mathrm{s}}=0.05 \mathrm{~m} \mathrm{~s}^{-1}$.

The sizes of $s_{0}, h_{0}, l_{0}, \zeta_{0}, \Psi_{0}, N_{0}, Q_{0}$ and $q_{0}$ have yet to be specified in the model. For an ice sheet, we can suppose $\Psi_{0}=10 \mathrm{~kg} \mathrm{~m}^{-2} \mathrm{~s}^{-2}$, and that a typical drainage length scale is $100 \mathrm{~km}$, i.e. $s_{0}=10^{5} \mathrm{~m}$. (It follows that $\tilde{\epsilon} \ll 1$.) As there are four relations in Equation (20) it is necessary to prescribe two other scales in order to determine the remaining ones. We put $N_{0}=1$ bar, based on borehole measurements on Ice Stream B, Antarctica (Engelhardt and Kamb, 1997). And since $\tilde{\epsilon} \ll 1$, it is natural to ensure in Equation (24) that $\mathcal{M}=O(1)$, by choosing an appropriate value of $[\mathcal{M}]$. Strictly speaking, this scale can be estimated from the basal-ice-melt rate and channel spacing, but the specification of these is difficult (particularly for ice sheets). Alternatively, one can simply prescribe $Q_{0}$ to be the (expected) outlet discharge. Here we use the nominal value $Q_{0}=$ $1 \mathrm{~m}^{3} \mathrm{~s}^{-1}$; then, the remaining scales are

$$
\begin{aligned}
& h_{0}=0.335 \mathrm{~m}, \quad l_{0}=5.77 \mathrm{~m}, \\
& \zeta_{0}=3.36 \times 10^{-2} \mathrm{~m}, \quad q_{0}=0.100 \mathrm{~m}^{3} \mathrm{~s}^{-1},
\end{aligned}
$$

and the corresponding parameters are

$$
\nu \approx 0.1, \quad \tilde{\epsilon} \approx 3.33 \times 10^{-3}, \quad r=0.9, \quad \tilde{\kappa} \approx 2.23 .
$$

Evidently, discharge contribution from roof melting is going to be negligible (since $\tilde{\epsilon} \ll 1$, independent of the choice of $N_{0}$ and $Q_{0}$ ), whereas the effect of till creep can be quite significant (since $\tilde{\kappa} \sim 1$ in Equation (25); remember however, that $\tilde{\kappa}$ is an upper bound estimate and depends on $N_{0}$ and $Q_{0}$ ). These inferences are unaffected if we had chosen $s_{0}$ according to the ice-sheet length scale $\left(\right.$ e.g. $\left.10^{3} \mathrm{~km}\right)$ instead.

\section{The reduced model}

By eliminating $h$ and $\zeta$ from Equations (21)-(27), we obtain, after some algebra,

$$
\begin{aligned}
& l=\left(\frac{\pi \sqrt{2} q}{I Q}\right)^{3 / 4} N_{\mathrm{c}}^{-n}, \\
& \Phi=C \frac{q^{3 / 2}}{Q^{5 / 2}} N_{\mathrm{c}}^{-n},
\end{aligned}
$$

and the following coupled model for $Q, q$ and $N_{\mathrm{c}}$ :

$$
\begin{aligned}
& \frac{\mathrm{d} Q}{\mathrm{~d} s}=\tilde{\epsilon} r C\left(\frac{q}{Q}\right)^{3 / 2} N_{\mathrm{c}}^{-n}+\mathcal{M}, \\
& \frac{\mathrm{d} q}{\mathrm{~d} s}=\frac{\tilde{\kappa}\left(1-n_{\mathrm{s}}\right) C}{N_{\infty}^{b}}\left(\frac{q}{Q}\right)^{3 / 2} N_{\mathrm{c}}^{a-2 n}+\mathcal{E}, \\
& \nu \frac{\mathrm{d} N_{\mathrm{c}}}{\mathrm{d} s}=\frac{C q^{3 / 2}}{Q^{5 / 2} N_{\mathrm{c}}^{n}}-\Psi .
\end{aligned}
$$

Here the constants are $I=\int_{-1}^{1}\left(1-X^{2}\right)^{7 / 6} \mathrm{~d} X \approx 1.275$ (by numerical integration) and $C=\pi^{5 / 2} / 2^{1 / 4} I^{3 / 2} \approx 10.2$.

Equation (35) describes the effective pressure distribution $N_{\mathrm{c}}(s)$ and is our drainage equation for a soft bed. This is supplemented by Equations (33) and (34) which provide recipes for $Q$ and $q$. Note that, if in our derivation we had neglected the sediment processes and imposed a cylindrical channel geometry (by putting $h=l$, dimensionally), then we would obtain

$$
Q\left(\Psi+\nu \frac{\mathrm{d} N_{\mathrm{c}}}{\mathrm{d} s}\right)^{7} \propto N_{\mathrm{c}}^{5 n},
$$

which is essentially the classical result (cf. Röthlisberger, 1972; equation (11)).

Given suitable boundary conditions, Equations (33)-(35) may be solved. We put $\Psi \equiv 1$, and for simplicity we assume $\mathcal{M}$ and $\mathcal{E}$ to be constants. These supply rates can be estimated from the typical discharge values expected at the snout. For instance, setting $\mathcal{M}=1$ (which gives $Q$ (snout) $\approx 1 \mathrm{~m}^{3} \mathrm{~s}^{-1}$ ) is consistent with our current model scaling, and as a first estimate, we can choose $\mathcal{E}=0.1$, such that the $\mathcal{E} / \mathcal{M}$ ratio corresponds to a (influx) suspended-sediment concentration of tens of grams per litre, typical of glacier-fed streams (Lawson, 1993). In general, the actual concentration in the channel would increase with downstream distance and exceed this, due to the effect of till creep ( since $\tilde{\kappa} \sim 1$ ). Our simulation results later confirm this, with the outflow-sediment load predicted at roughly $100 \mathrm{~g} \mathrm{~L}^{-1}$, which is still within the plausible range.

We also specify a nominal (dimensional) value $N_{\infty}=$ 
1 bar. The parameters in Equations (33) and (34) are then $\tilde{\epsilon} r C \approx 0.03$ and $\tilde{\kappa}\left(1-n_{\mathrm{s}}\right) C N_{\infty}^{-b} \approx 16$ (an upper bound estimate). These values indicate that $Q$ is an increasing function of $s$, with $\mathrm{d} Q / \mathrm{d} s \approx \mathcal{M}$, whereas $q(s)$ also increases monotonically, but not necessarily linearly.

The solution for $N_{\mathrm{c}}$ is more complicated. $\nu \ll 1$ suggests that Equation (35) may be approximated as

$$
N_{\mathrm{c}}^{n}=\frac{C q^{3 / 2}}{\Psi Q^{5 / 2}}
$$

which indicates a canal-type characteristic, i.e. $N_{\mathrm{c}} \propto Q^{\beta}$, where $\beta=-5 / 2 n<0$ (and $\beta=-5 / 6$ with $n=3$ ). However, this is a singular approximation because $\nu$ multiplies into a first-order derivative. There will be a boundary layer (of thickness $O(\nu)$ corresponding to a distance of $10 \mathrm{~km}$ ) in which effective pressure gradients are significant, and this occurs at the head of the stream. Nevertheless, the canal drainage law (Equation (37)) is applicable outside this boundary layer, sufficiently far downstream that pressure gradients are negligible.

Last, we discuss the form of the boundary conditions at $s=0$. Care is required here in order to avoid the righthand terms in Equations (33)-(35) becoming singular, which will occur if $N_{\mathrm{c}}=0$ or $Q=0$ there. Our prescription is based on the following consideration. Although the channel has its top end defined at $s=0$, we envisage that it is connected to (Darcy) drainage in the till via some kind of channel filaments, in which the water flux is small but non-zero; hence, $Q$ should not vanish there, or $Q(0)>0$. On the other hand we specify $q(0)=0$, by assuming that the incipient discharge would be too small to sustain sediment transport, i.e. the flow stress at the bed $(\approx \tau / 2)$ there is below its critical value for sediment entrainment. As a result, we can also expect these incipient channels to behave essentially like hard-bed channels, with $N_{\text {c }}$ directly related to $Q$ (Röthlisberger, 1972). Therefore $N_{\mathrm{c}}(0)$ should be relatively small (but non-zero, since that would imply flotation of the ice), and a natural choice is $N_{\mathrm{c}}(0)=N_{\infty}$. Given these boundary conditions, a local analysis of the model shows that the solutions are well-behaved, and in particular neither $N_{\mathrm{c}}$ nor $Q$ would vanish in $0 \leq s \leq 1$.

\section{Numerical solution}

Equations (33)-(35) have been solved by using a simple Euler predictor-corrector algorithm. This algorithm uses finite difference and steps forwards in $s$ from $s=0$, where we specify the dimensionless initial conditions $Q(0)=10^{-2}$ (corresponding to $0.01 \mathrm{~m}^{3} \mathrm{~s}^{-1}, \sim Q$ (snout)/100), $q(0)=0$, and $N_{\mathrm{c}}(0)=1$ (corresponding to 1 bar). As a trial simulation, we employ the scales and parameters given in Equations (29) and (30), and also the model constants $\mathcal{M}=1, \mathcal{E}=0.1, \Psi=1$.

Figure 2 shows the computed results plotted in dimensioned units. As expected, we see the downstream increase of $Q$ and $q$ (Fig. 2a) and the boundary-layer behaviour in $N_{\text {c }}$ (Fig. 2b). The canal solution in Equation (37) has been included for comparison, and is found to be a good approximation in $s \gtrsim 30 \mathrm{~km}$, where the predicted effective pressure is $N_{\mathrm{c}} \approx 1.5$ bar. We have also investigated the effect of varying $N_{\mathrm{c}}(0)$ in the simulation. Downstream in the "canal" region, $N_{\mathrm{c}}$ is insensitive to its boundary value at the stream head (Fig. 2c).

Figure 3 shows other results derived from Figure $2 \mathrm{a}$ and b. The suspended-sediment load in $\mathrm{g} \mathrm{L}^{-1}$ is calculated by evaluating $\rho_{\mathrm{s}} q / Q$ (Fig. 3a), and is found to be of the order of
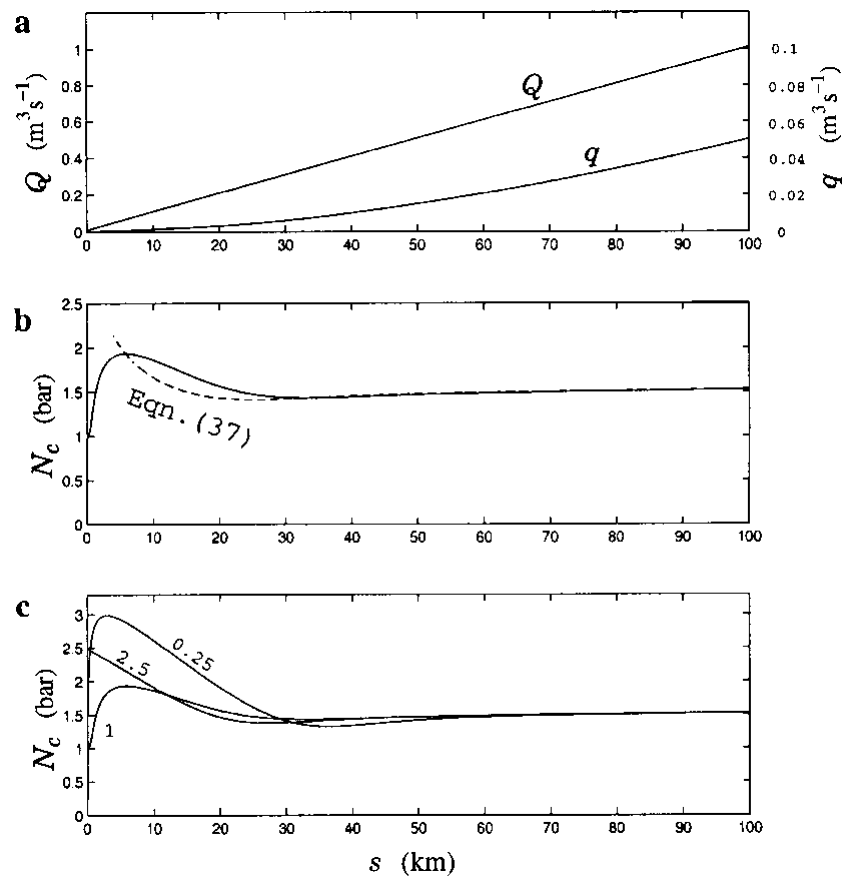

Fig. 2. (a) Computed discharge, $Q$, and sediment flux, $q$, and (b) computed effective channel pressure, $N_{\mathrm{c}}$, as functions of downstream distance, s. Dashed line represents $N_{\mathrm{c}}$ as given by Equation (37). (c) Sensitivity of the simulated $N_{\mathrm{c}}(s)$ to its upstream boundary condition. Test values are $N_{\mathrm{c}}(0)=$ $0.25,1$ (as in (b)), and 2.5 bar.

$100 \mathrm{~g} \mathrm{~L}^{-1}$, rather high in the plausible range of values. This is due to the large (upper-bound) value of $\tilde{\kappa}$ used in the simulation. For example, reducing $\tilde{\kappa}$ by a factor of ten would reduce the sediment load at the outlet to about $50 \mathrm{~g} \mathrm{~L}^{-1}$. In addition, we have calculated the depth and width variations
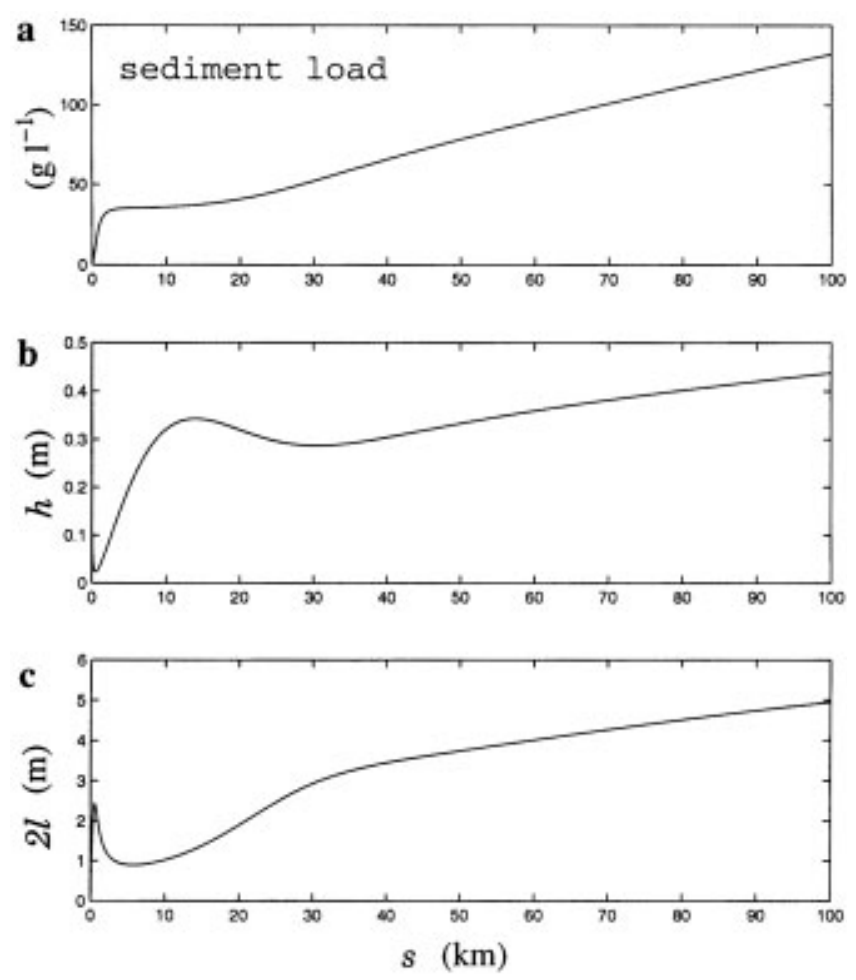

Fig. 3. Downstream variations of (a) sediment load, (b) channel depth $h(x=0, s)$ and (c) total channel width $2 \times l(s)$, corresponding to the simulation in Figure $2 a$ and $b$. 
of the channel $h(x=0, s)$ and $l(s)$ via Equations (22) and (31) (see Fig. 3b and c). In general $h \ll 2 l$, so the channel adopts a wide cross section, consistent with model assumptions. ( $h$ appears to blow up close to $s=0$. This spurious feature is due to the neglect of bedload transport in the model, and does not affect the canal approximation downstream. Further examination of this is given by $\mathrm{Ng}$ (manuscript in preparation).) At the snout, the channel cross section is approximately $5 \mathrm{~m}$ across by $0.45 \mathrm{~m}$ deep.

Finally, our solution (and Equation (37)) indicates a nonzero value of $N_{\mathrm{c}}$ at $s=100 \mathrm{~km}$. This is significant because if the ice thickness $\left(H_{\mathrm{i}}\right)$ vanishes at the snout then the channel water pressure $\left(p_{\mathrm{c}}\right)$ would become negative $(!)$ there. In practice, we suppose open-channel flow occurs when $p_{\mathrm{c}}=0$, so our model predicts that pressurized flow would terminate some distance back from the snout. The position of this transition $s=s_{\mathrm{co}}$ is given approximately by the relation

$$
N_{\mathrm{c}}\left(s_{\mathrm{co}}\right)=\rho_{\mathrm{i}} g H_{\mathrm{i}}\left(s_{\mathrm{co}}\right) .
$$

For our model ice sheet, $H_{\mathrm{i}}\left(s_{\mathrm{co}}\right)$ is about $20 \mathrm{~m}$.

\section{DISCUSSION}

The present work furthers our understanding of soft-bed subglacial channels and exposes some of the limitations of previous models. Where ice overrides till, the coupled flow of water and sediment is inevitable, and any realistic theory of soft-bed drainage must take this into account. We have developed a model which supersedes that of Walder and Fowler (1994) and our findings support their conclusion that sediment-floored channels can exhibit canal-type characteristics, with effective pressure inversely related to discharge; the exact relation depends on the channel sediment flux. This behaviour, summarized by Equation (37), occurs where effective pressure gradients are small and is independent of the value assumed for the constant $n$ in Glen's flow law, as long as $n>0$.

Regarding downstream variation, it is interesting to compare the predictions of our theory and the classical theory (Röthlisberger, 1972), where sediment processes are ruled out altogether and restriction of a cylindrical-channel cross section is enforced. The main difference lies in the form of the respective drainage equations. Written in the form of Equation (35) (which applies for a canal), Equation (36) (for an R channel) would have a positive power of $N_{\mathrm{c}}$ appearing in the numerator of the first righthand term. The implication is that $N_{\text {c }}$ can decrease to a small value only with negative slope $\left(\mathrm{d} N_{\mathrm{c}} / \mathrm{d} s<0\right)$. For instance, taking $N_{\mathrm{c}}$ (snout) $=0$ (the boundary condition used by Röthlisberger) and a linearly increasing distribution for $Q$, it is straightforward to confirm this by integrating Equation (36) numerically. In this case, the boundary layer in $N_{\text {c }}$ occurs near the channel outlet, with $N_{\text {c }}$ reaching a near-constant (high) value upstream. This is essentially the opposite result to that for the canal, where $N_{\text {c }}$ may assume any non-zero (but presumably low) till value at the stream head because the boundary layer occurs there (see Fig. 2b and c); in this case however, $N_{\mathrm{c}}$ cannot vanish at the outlet, so the canal model actually predicts the position of the closed/open-channel flow transition near the snout. (Although Röthlisberger's (1972) model does allow the possibility of open-channel flow under some conditions, he defined the channel outlet to be situated where the flow transition takes place, without specifying its position relative to the snout; see p. 178 and 185.)

Our theory can be extended to a more general description for soft-bed drainage. Notably, it provides a starting point for investigating how the canals would couple to each other and to other forms of drainage. Currently, the quantities $N_{\mathrm{c}}(0), Q(0), \mathcal{M}(s)$ and $\mathcal{E}(s)$ are poorly constrained in the model. The question of how to specify these is intimately related to the problem of determining the architecture of the drainage network.

For instance, one can consider a network in which the tributary channels feeding our main channel are themselves sustained by finer tributaries, and so on; each level of the hierarchy may be described by our ingredient model. In building a model for the entire drainage system, the four quantities in question would then appear as unknowns, instead of being externally prescribed. In particular, $N_{\mathrm{c}}(0)$ and $Q(0)$ would describe how the canals are connected to the till via incipient channels, and in isolated cases, to englacial sources; $\mathcal{M}$ and $\mathcal{E}$ would describe the network structure (channel spacing and order of linkage). Model solution determines the system architecture, the basal-water-pressure distribution, and in addition, the rate at which till sediments are depleted by drainage. The last of these is relevant to the sediment budget and is clearly important in the long term, as changes of till thickness could have a drastic effect on the nature of basal-ice dynamics. This generalized model awaits further investigation.

\section{AGKNOWLEDGEMENTS}

The author thanks St. John's College, University of Oxford for a Junior Research Fellowship position. A. C. Fowler continues to be a constant source of inspiration.

\section{REFERENCES}

Alley, R. B. 1989. Water-pressure coupling of sliding and bed deformation: I. Water system. F. Glaciol., 35(119), 108-118.

Alley, R. B., D. D. Blankenship, C. R. Bentley and S. T. Rooney. 1986. Deformation of till beneath Ice Stream B, West Antarctica. Nature, 322(6074), 57-59.

Alley, R. B., D. D. Blankenship, C. R. Bentley and S.T. Rooney. 1987. Till beneath Ice Stream B. 3. Till deformation: evidence and implications. f. Geophys. Res., 92(B9), 8921-8929.

Boulton, G. S. and R. C. A. Hindmarsh. 1987. Sediment deformation beneath glaciers: rheology and geological consequences. 7. Geophys. Res., 92(B9), 9059-9082.

Engelhardt, H. and B. Kamb. 1997. Basal hydraulic system of a West Antarctic ice stream: constraints from borehole observations. F. Glaciol., 43(144), 207-230.

Fowler, A. C. and C. Johnson. 1995. Hydraulic run-away: a mechanism for thermally regulated surges of ice sheets. F. Glaciol., 41 (139), 554-561.

Fowler, A. C. and C. Johnson. 1996. Ice-sheet surging and ice-stream formation. Ann. Glaciol., 23, 68-73.

Fowler, A. C. and F.S.L. Ng. 1996. The role of sediment transport in the mechanics of jökulhlaups. Ann. Glaciol., 22, 255-259.

Fowler, A. C. and E. Schiavi. 1998. A theory of ice-sheet surges. F. Glaciol., 44(146), 104-118.

Iverson, N. R., B. Hanson, R. LeB. Hooke and P. Jansson. 1995. Flow mechanism of glaciers on soft beds. Science, 267(5194), 80-81.

Iverson, N. R., T. S. Hooyer and R. W. Baker. 1998. Ring-shear studies of till deformation: Coulomb-plastic behavior and distributed strain in glacier beds. f. Glaciol., 44(148), 634-642.

Kamb, B. 1991. Rheological nonlinearity and flow instability in the deforming bed mechanism of ice stream motion. 7. Geophys. Res., 96(B10), $16,585-16,595$.

Lawson, D. E. 1993. Glaciohydrologic and glaciohydraulic effects on runoff and sediment yield in glacierized basins. CRREL Monogr. 93-02.

$\mathrm{Ng}$, F. S. L. 1997. A mathematical model of wide subglacial water drainage 
channels. InWettlaufer, J. S., J. G. Dash and N. Untersteiner, eds. Ice physics and the natural environment. Berlin, etc., Springer-Verlag, 325-327. (NATO ASI Series I: Global Environmental Change 56.)

Nye, J. F. 1953. The flow law of ice from measurements in glacier tunnels, laboratory experiments and the Jungfraufirn borehole experiment. Proc. R. Soc. London, Ser. A, 219(1139), 477-489.

Parker, G. 1978. Self-formed straight rivers with equilibrium banks and mobile bed. Part 1: The sand-silt river. F. Fluid Mech., 89(1), 109-125.
Richards, K. 1982. Rivers: form and process in alluvial channels. New York, Methuen and Co.

Röthlisberger, H. 1972. Water pressure in intra- and subglacial channels. $f$. Glaciol., 11 (62), 177-203.

Shoemaker, E. M. 1986. Subglacial hydrology for an ice sheet resting on a deformable aquifer. F. Glaciol., 32(110), 20-30.

Walder, J. S. and A. Fowler. 1994. Channelized subglacial drainage over a deformable bed. F. Glaciol., 40(134), 3-15. 